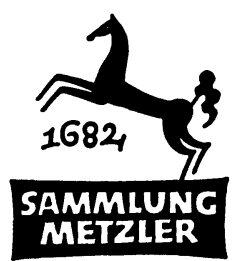

REALIEN ZUR LITERATUR

ABT. D:

LITERATURGESCHICHTE 


\section{Der deutsche Bildungsroman}


CIP-Kurztitelaufnahme der Deutschen Bibliothek

Selbmann, Rolf:

Der deutsche Bildungsroman / Rolf Selbmann. Stuttgart: Metzler, 1984.

(Sammlung Metzler; M 214: Abt. D,

Literaturgeschichte)

ISBN 978-3-476-10214-0

NE: GT

ISBN 978-3-476-10214-0

ISBN 978-3-476-03918-7 (eBook)

DOI 10.1007/978-3-476-03918-7

\section{214}

(C) 1984 Springer-Verlag GmbH Deutschland

Ursprünglich erschienen bei J. B. Metzlersche Verlagsbuchhandlung und Karl Ernst Poeschel Verlag GmbH in Stuttgart 1984 


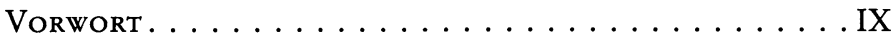

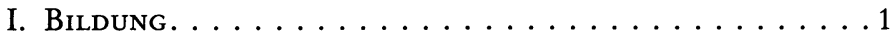

1. Die Entstehung der Bildungsidee ...............

2. Wandlungen des Bildungsbegriffs $\ldots \ldots \ldots \ldots \ldots \ldots$

II. Der Begriff »Bildungsroman « . . . . . . . . . . . 9

1. Historische Bestimmungen. Von Blanckenburg bis Hegel . . .9

2. Die literaturwissenschaftliche Diskussion ..........18

III. Versuch einer Gattungsbestimmung . . . . . . . . . 34

1. Zur Problematik der Gattung »Bildungsroman «........34

2. Bildungsstruktur, Bildungsgeschichte, Bildungsroman. ...38

IV. Die Entstehung des Bilddungsromans . . . . . . . . . 42

1. Die Bildungsgeschichte im Roman des 18. Jahrhunderts . . .42 Schummel: Empfindsame Reisen durch Deutschland Knigge: Geschichte Peter Clausens - Hegrad: Komischer Roman - Wieland: Geschichte des Agathon

2. Der Erzähler und sein Held. Autobiographie und Bildungsgeschichte ..........................52 Zur modernen Autobiographie - Moritz: Anton Reiser

V. »Wilhelm Meister « Als Muster der Gattung . . . . . 63

1. Goethes Roman als Bildungsroman . ...........63 Bildung im "Wilhelm Meister « - Wilhelm Meisters Bildungsgeschichte - Bildungsgeschichte und Gesellschaftsstruktur

2. Gegenmodelle.......................82 Jean Paul: Titan - Hölderlin: Hyperion, Novalis: Heinrich von Ofterdingen - Eichendorff: Ahnung und Gegenwart - Hoffmann: Kater Murr

VI. Der Bildungsroman im i9. Jahrhundert . . . . . . . 102

1. Zwischen Individualroman und Gesellschaftsroman. . . . 102 Mörike: Maler Nolten - Tieck: Der junge Tischlermeister - Immermann: Die Epigonen

2. Der Bildungsroman nach $1848 \ldots \ldots \ldots \ldots \ldots \ldots 129$

Arbeit im Bildungsroman - Keller: Der grüne Heinrich

- Stifter: Der Nachsommer - Raabe 
VII. Ausblick: Der Bildungsroman im 20. Jahrhundert . 153

1. Zwischen Ideologie und Parodie . . . . . . . . . . 153

2. Spuren des Bildungsromans in der Gegenwart ....... 158

NAmen- und Titelregister . . . . . . . . . . . . . 160 
Akten des VI. Internationalen Germanisten-Kongresses Basel 1980. Teil 3. Hrsg. v. Heinz Rupp und Hans-Gert Roloff. Bern, Frankfurt, Las Vegas 1980 (= Jahrbuch für Internationale Germanistik. Reihe A. Kongreßberichte. Band 8).

AR Karl Philipp Moritz: Anton Reiser. Ein psychologischer Roman. Mit Textvarianten, Erläuterungen u. einem Nachwort hrsg. v. Wolfgang Martens. Stuttgart 1972.

AuG Freiherr Joseph von Eichendorff: Sämtliche Werke. Hist.Krit. Ausgabe hrsg. v. Wilhelm Kosch und August Sauer. Regensburg o. J. Band 3: Ahnung und Gegenwart.

DR Der deutsche Roman. Vom Barock bis zur Gegenwart. Hrsg. v. Benno v. Wiese. Düsseldorf 1963.

DVjs Deutsche Vierteljahrsschrift für Literaturwissenschaft und Geistesgeschichte.

E Karl Immermann: Die Epigonen. Familienroman in neun Büchern 1823-1835. (= Werke in 5 Bänden. Hrsg. v. Benno v. Wiese. 2. Band. Frankfurt 1971)

FdL Formen der Literatur in Einzeldarstellungen. Hrsg. v. Otto Knörrich. Stuttgart 1981. (= Kröners Taschenausgabe 478)

gH Gottfried Keller: Sämtliche Werke und ausgewählte Briefe. Hrsg. v. Clemens Heselhaus. München 1968. Band 1: Der grüne Heinrich.

I Interpretationen III. Deutsche Romane von Grimmelshausen bis Musil. Hrsg. v. Jost Schillemeit. Frankfurt 1966.

KR Friedrich Hegrad: Komischer Roman. 2 Theile. Leipzig und Wien 1786.

L Johann Wolfgang von Goethe: Werke. Berliner Ausgabe. Berlin 1976. Band 10: Wilhelm Meisters Lehrjahre. Literaturwissenschaft und Sozialwissenschaften. Stuttgart. E. T. A. Hoffmann: Die Elixiere des Teufels/Lebens-Ansichten des Katers Murr. München 1969 (= Werke in Einzelausgaben 2).

N Eduard Mörike: Maler Nolten. Novelle. Hrsg. v. Herbert Meyer. Stuttgart 1967 (= Hist.-Krit. Gesamtausgabe 3).

Ns Adalbert Stifter: Der Nachsommer. Eine Erzählung. Hrsg. v. Max Stefl. Augsburg 1954 (= Ausgabe in Einzelbänden 7).

PC Adolph Freiherr Knigge: Geschichte Peter Clausens. Zweyte, von dem Verfasser verbesserte Auflage. Frankfurt 1794. Reprint Frankfurt 1971.

RBR Romane und Erzählungen des Bürgerlichen Realismus. Neue Interpretationen. Hrsg. v. Horst Denkler. Stuttgart 1980.

RER Romane und Erzählungen der deutschen Romantik. Neue Interpretationen. Hrsg. v. Paul Michael Lützeler. Stuttgart 1981. 
RRR Romane und Erzählungen zwischen Romantik und Realismus. Neue Interpretationen. Hrsg. v. Paul Michael Lützeler. Stuttgart 1983.

Sch $\quad$ Friedrich Schiller: Werke. Nationalausgabe. Weimar 1962. 20. Band: Philosophische Schriften, 1. Teil.

T Jean Paul: Werke in zwölf Bänden. Hrsg. v. Norbert Miller. München 1975. Band 5 und 6: Titan.

Tm Ludwig Tieck: Werke in vier Bänden. Hrsg. v. Marianne Thalmann. München 1975. Band 4: Romane, darin: Der junge Tischlermeister.

VASILO Vierteljahresschrift des Adalbert-Stifter-Instituts des Landes Oberösterreich.

$\mathrm{WdF}$

Wege der Forschung. Darmstadt.

Wj

Johann Wolfgang von Goethe: Werke. Berliner Ausgabe. Berlin 1976. Band 11: Wilhelm Meisters Wanderjahre.

$\mathrm{ZfdPh} \quad$ Zeitschrift für deutsche Philologie. 
Der vorliegende Band zum deutschen Bildungsroman leitet seine Existenzberechtigung aus einem eklatanten Mangel des literaturwissenschaftlichen Betriebs her: bis heute gibt es keine brauchbare Gattungsgeschichte des Bildungsromans oder ein Handbuch, das diesen Namen verdient. Eine Handreichung, die eine Auseinandersetzung mit einem der zentralen Probleme der neueren Literaturwissenschaft ermöglicht, indem sie wichtige Fragen wenigstens anreißt und die unübersehbare Literatur sichtet, einordnet und aufschlüsselt, ist also ein Desiderat. Diesem Bedürfnis nach Übersichtlichkeit hat die Darstellung durch den Mut zur Kürze gerecht zu werden. In der Verbindung von systematischem und literaturgeschichtlichem, d. h. chronologischem Vorgehen kam es darauf an, die Geschichte der Gattung zugleich als Entwicklungsprozeß einer Textreihe und mit Blick auf die Systematik der Gattungstheorie vorzustellen. Schon im ersten Zugriff auf die Themenstellung war eine merkwürdige Beobachtung bei der Verwendung des Begriffs Bildungsroman aufzulösen, die Tatsache nämlich, daß der Terminus als verfestigter, allgemein akzeptierter Begriff verwendet wird, obwohl gleichzeitig so gut wie jeder Bildungsroman des 18. und 19. Jh.s in dieser Zuschreibung umstritten ist. Mehr noch: was denn nun ein richtiger Bildungsroman sei - diese Frage ist für jeden Roman und in jeder Epoche ganz unterschiedlich beantwortet worden. Mit dieser Geschichtlichkeit nicht nur der Gattung, sondern auch ihrer Interpretation hängt schließlich zusammen, daß die Literaturwissenschaft mit dem deutschen Bildungsroman ein doppelt heißes Eisen vor sich hat. Sicher gibt es gute Gründe, Gattung und Begriff des Bildungsromans auf den nationalliterarischen Aspekt zu beschränken und die Romanart als spezielle deutsche Ausprägung der europäischen Erzählkunst seit der Aufklärung zu verstehen. Doch genau an dieser Stelle beginnt auch die Ideologisierung, ja Fetischisierung der Gattung zur deutschen Romanform schlechthin:

"eine Romanart, die ein ganz ausgesprochen nationales Gepräge trägt, wie sie eigenartiger, individueller kein anderes Volk aufzuweisen hat, den deutschen Bildungsroman, der im letzten Jahrhundert ganz eigentlich der Roman der Dichter und Denker war und es voraussichtlich auch bleiben wird.« (H. A. Krüger, 1906)

Solche Redeweisen, und die genannte ist nicht die schlimmste, lassen eine kritische Untersuchung, was denn nun das besonders Deutsche dieser Gattung sei, nicht mehr zu. Hier ziehen sich die Interpreten 
mit ihren Texten in die machtgeschützte Innerlichkeit deutscher Wesensart zwischen germanistischem Elfenbeinturm und völkischem Sendungsbewußtsein zurück. Auf der anderen Seite ist es wohl wahr, daß die Gattungsgeschichte des deutschen Bildungsromans seit etlichen Jahrzehnten zum wissenschaftsmethodischen Prüfstein geworden ist, an dem sich jede neu entwickelte Gattungstheorie messen lassen muß. Es ist kein Zufall, daß der Bildungsroman - so oder so - in vieler Munde ist, noch bevor man sich geeinigt hat, wovon man denn eigentlich spricht. Als Realienbuch versucht die vorliegende Darstellung deshalb, einen Mittelweg zwischen der Beschreibung von Gattungsdeterminanten und expliziten Romaninterpretationen einzuschlagen. Bei letzteren war hauptsächlich die Systematisierung von Detailuntersuchungen zu leisten; nur gelegentlich wurden neue Textzugänge aufgezeigt, immer aber, wo es nötig schien, Forschungsschwerpunkte hervorgehoben. Hingegen werden manche Aspekte der einzelnen Romane, wenn sie außerhalb der Gattungsproblematik liegen und von der Forschung genügend beackert worden sind, vielfach nur genannt. Mit Hilfe der weiterführenden Literatur kann sich der spezieller interessierte Leser jederzeit leicht einarbeiten. Die wichtigsten Texte zur Theorie des Bildungsromans und seiner Erforschung sind leicht zugänglich in dem von mir herausgegebenen Wege-der-Forschung-Band »Der deutsche Bildungsroman« (voraussichtlich 1985).

München, im März 1984

R.S. 